\title{
INCLUSION SOCIAL Y PARTICIPACION COMUNITARIA: UNA PERSPECTIVA DE TRABAJO FRENTE A LA DISCAPACIDAD
}

\author{
SOCIAL INCLUSION AND COMMUNITY PARTICIPATION: \\ AN ALTERNATIVE WORK IN FRONT DISABILITY
}

\author{
Alejandra Alvarado* \\ Maria Elisa Moreno** \\ Maria Clara RodrigueZ ${ }^{* * *}$
}

\begin{abstract}
RESUMEN
Estudio de investigación acción participativa que tuvo como objetivo desarrollar y evaluar, junto con la comunidad, la percepción del programa fundamentado en un modelo socioecológico, con el fin de promover la inclusión social de las personas en situación de discapacidad y sus familias residentes en una comunidad del municipio de Chía en Colombia, desde el desarrollo personal, familiar y comunitario. Inicialmente se identificaron los significados que alrededor de la discapacidad se han construido, los cuales están relacionados con los problemas que deben afrontar en su cotidianidad y como una condición que les permite reconocer su valía y sus potencialidades. En el proceso de construcción de este modelo participaron 14 personas en situación de discapacidad, sus cuidadores, 8 líderes comunitarios, 6 miembros de la Junta de Acción Comunal y un equipo conformado por profesionales de enfermería, educación y psicología. La identificación de los recursos disponibles se llevó a cabo a través de foros comunitarios y el uso de estrategias como las metáforas del árbol y del sol. Los resultados muestran que el desarrollo personal y familiar, se logra desde la percepción de bienestar y respaldo; el desarrollo comunitario desde el apoyo social, la satisfacción de necesidades, la identificación y la generación de recursos
\end{abstract}

Palabras clave: Modelo socioecológico, inclusión social, personas con discapacidad, participación comunitaria.

\begin{abstract}
The purpose of this participative action research was to develop and evaluate with the community, the perception of a program based on a socio ecological model for the social inclusion of people with disabilities and their families who live in a community of the municipality of Chia, in Colombia. The project was developed in three phases. During the planning phase, the researchers identified the meanings of "disability condition" that are given by the patients, their families and the support networks and it was found that these were related to the difficulties disabled people and their families must face in their daily living and how this condition might allow them to recognize their sheer value and potential. During the second phase of action and the third phase of evaluation, 14 people with disabilities, their caregivers, 14 community leaders and a team formed by nursing, psychology and education professionals were involved in the construction and development of the model. The identification of available resources was carried out through interviews, focal groups and community forums using strategies such as the tree and the sun metaphors. The results show that the personal and family development nucleus is accomplished from the perception of welfare and support. The community development nucleus is accomplished from the social support, the satisfaction of needs, and the identification of resources and resource generation.
\end{abstract}

Keywords: Socio ecological model, social inclusion, disabled persons, communitary participation

Fecha recepción: 10/04/08 Fecha aceptación: 16/03/09

\footnotetext{
*Enfermera, Magíster en Enfermería, profesora asistente Facultad de Enfermería y Rehabilitación, Universidad de La Sabana, Alejandra.alvarado@unisabana.edu.co

${ }^{* *}$ Enfermera, Especialista en Enfermería en cardiorrespiratorio, profesora asociada, Facultad de Enfermería y Rehabilitación, Universidad de La Sabana, mariae.moreno@unisabana.edu.co

${ }^{* *}$ Psicóloga, Magíster en Investigación, profesora asociada Facultad de Psicología, Universidad de La Sabana,mariarb@unisabana.edu.co
} 


\section{INTRODUCCIÓN}

La discapacidad ha sido, en los últimos años, un tema de interés no sólo para los profesionales de la salud sino para las instituciones que tienen la responsabilidad de dar respuesta a las necesidades de estas personas. Lo anterior se fundamenta en las estadísticas que a través de los años han marcado tendencias que no son del todo subestimables, más aún cuando el último censo del Departamento Nacional de Estadística en Colombia(1) arroja una cifra en la que, de cada 100 colombianos, 6.3 (segundo país en Latinoamérica después de Brasil) tienen una limitación permanente, con unas condiciones de restricción que impactan la satisfacción de sus necesidades básicas. Por ello las metas del cuidado de enfermería se centran en ayudar a las personas a utilizar al máximo sus capacidades residuales, a mantener un óptimo estado de salud y a adaptarse a un estilo de vida que se modificó por la nueva condición(2).

Este cuidado de enfermería tradicionalmente se ofrecía en los centros hospitalarios y servicios de rehabilitación. Sin embargo, los costos de las estancias prolongadas y las complicaciones que de éstas se derivaban en términos de adaptación e inclusión social de las personas con discapacidad, motivaron el desarrollo de políticas encaminadas a promover el alta temprana y desarrollar estrategias que propendieran por la reintegración de las personas a la comunidad. De tal manera que los ámbitos del cuidado se modificaron y generaron la necesidad de desarrollar nuevos servicios para la atención y rehabilitación de estas personas.

Esta primera reflexión nos sitúa frente a una problemática, que ha sido reconocida por los diferentes estados y su respuesta ha sido acorde a su sistema sociopolítico y económico. La variabilidad en las formas de atención se vinculan y se evidencian en los planes de seguridad social como un servicio que ofrece garantías en relación con programas complementarios pero que a la vez impone restricciones, según las reglamentaciones vigentes.
La complejidad de las situaciones que se presentan en la atención de las personas con discapacidad y sus familias, no puede ser abordada por una sola disciplina, requieren del trabajo en equipos interdisciplinarios, el cual se caracteriza por la cooperación para alcanzar las metas propuestas. Este tipo de trabajo exige una sólida formación disciplinar en cada uno de los integrantes, de tal forma que conocen el dominio de su profesión y su aporte individual al logro de una meta común que es la rehabilitación del paciente y la inclusión social.

Si bien el conocimiento disciplinar es fundamental, a la hora de establecer un programa, es esencial que los miembros estén de acuerdo en abordar la problemática desde una perspectiva teórica común. Los programas que existen en la actualidad se enmarcan en modelos de rehabilitación como los centrados en síntomas, que se desarrollan en centros hospitalarios y de educación especial; el de rehabilitación basada en la comunidad, centrado en el modelo medico de promoción, prevención, tratamiento y rehabilitación, con participación comunitaria $(3,4)$.

En este sentido, hoy el desarrollo de políticas y programas se convierte en un eje de interés sobre el cual se deben orientar las acciones de investigación, en las cuales la participación de la comunidad permita avanzar en los diversos campos para que las personas en condición de discapacidad puedan lograr los mismos niveles de calidad de vida que aquellas que no se encuentran en esta situación y de esta manera minimizar la exclusión social. Zabriskei, Lundberg y Groff(5) investigaron las inequidades a las que se veían sometidas las personas con discapacidad. Ellos encontraron que los indicadores relacionados con calidad de vida, tales como la salud, la educación, el empleo, la participación política, la recreación y la satisfacción general con la vida eran tan bajos, que las propuestas deberían dirigirse a lograr el bienestar desde las áreas del deporte y la actividad física, que habían sido documentadas para la población que no tenía discapacidad.

Igualmente, Zabriskei y Heine(6) lograron mejorar la calidad de vida de las familias a 
través de la recreación terapéutica. Los argumentos están relacionados con las posibilidades de interacción social y las oportunidades de retroalimentación. Sin embargo, aunque los resultados obtenidos son importantes, Moreno(7) argumenta la necesidad de tomar como punto de partida los modelos sociales ya que éstos reclaman la responsabilidad del entorno en el proceso de inclusión social. Esto, históricamente, se ha combatido con educación, legislación y política(8).

Otros problemas como las altas tasas de desempleo y la necesidad de supervisión familiar y comunitaria y una baja participación social, han sido característicos de esta población. Su intervención desde el acompañamiento para el desarrollo de habilidades de interacción en ambientes naturales ha mejorado la participación comunitaria y la percepción sobre una calidad de vida satisfactoria, lo que significa un incremento en la independencia en el manejo del hogar y la participación en actividades productivas para los participantes(9). Por otro lado el análisis de la discapacidad desde la teoría de sistemas permitió un trabajo más abarcador al incluir otras dimensiones como la cultural y la social. Esta teoría reconoció que todos los elementos del fenómeno están íntimamente relacionados entre sí y que las respuestas expresan la totalidad del mismo. Este nuevo enfoque propició el desarrollo de programas de rehabilitación en comunidad como una metodología de trabajo que permitía integrar de manera coordinada los diferentes actores sociales (personas con discapacidad, familia, escuela, empleadores, estado y sociedad civil) en la búsqueda de alternativas orientadas a la integración social de las personas con discapacidad, a través de la participación activa de la sociedad y tomando en cuenta los recursos existentes(10). De los programas de rehabilitación basados en la comunidad que se llevan a cabo en Colombia, vale la pena destacar los trabajos que se están realizando en algunas regiones del país, como el que se desarrolla en la Costa Pacífica(11), el programa del Comité Regional de Rehabilitación de Antioquia y en otras regiones colombianas que han sido fundamentados en una perspectiva ecológica de la discapacidad.

Dentro de esta línea de trabajo, son muchas las investigaciones que han dado cuenta de la eficacia de seguir este camino. Vitztum Komanecki(12) por ejemplo, argumentó que la integración comunitaria es una meta importante para la inclusión social en la niñez. Otra alternativa de integración a la comunidad como la desinstitucionalización ha resultado en una rápida expansión de servicios para las personas que están en condiciones de discapacidad. En la Universidad de Queens en Kingston, Blessing (2004) se utilizó esta estrategia elaborada desde un marco de aculturación y encontró que era posible la integración en la medida en que las personas se vincularan y fueran apoyadas y retroalimentadas sobre el éxito en su productividad(13).

Los trabajos de investigación revisados hasta el momento muestran que es posible avanzar en la inclusión social, en la medida en que se logren identificar los factores asociados a la exclusión y a la vez diseñar propuestas que busquen su solución. En este sentido se establece el acompañamiento fundamental en estos procesos de cambio, pero a la vez se reclama por un trabajo centrado en un paradigma más abarcador, en el que el interés por comprender a la persona en su interacción con el entorno incluya el análisis de los diversos componentes de la ecología humana.

Uno de los teóricos más reconocidos en el marco de la ecología humana es Urie Bronfrenbrenner(14), autor del Modelo Socioecológico, quien reconoce que en el abordaje de una situación, el investigador no sólo debe entender las entidades en aislamiento, sino también la relación entre ellas. El desarrollo individual debe comprenderse en el contexto del ecosistema, así un individuo crece y se adapta a través del intercambio con su ambiente inmediato (la familia) y ambientes más distantes como la escuela y la comunidad.

En efecto, la comunidad se constituye en una oportunidad para la inclusión, en la medi- 
da en que desde ella se promueven y estimulan las políticas que dan sentido y permean las vidas de los individuos, para garantizar que las barreras que la sociedad ha creado alrededor de la discapacidad sean eliminadas. La propuesta de Schuller(15) encaja en este sentido, al considerar las desventajas que deben afrontar las personas con discapacidad, incluyendo el alto riesgo de victimización. A su juicio, la inclusión como posibilidad se construye desde el trabajo con los agentes sociales, quienes a través de la actualización permanente de las necesidades, podrán acceder y proporcionar las bases para el cambio.

\section{Objetivo general}

Desarrollar y evaluar, junto con la comunidad, la percepción del programa fundamentado en un modelo socioecológico, con el fin de promover la inclusión social de las personas en situación de discapacidad y sus familias, desde el desarrollo personal, familiar y comunitario.

\section{MATERIALES Y MÉTODO}

Se utilizó un abordaje cualitativo de investigación-acción participativa. Este es un método sistemático e intencional de indagación que utilizan los investigadores para reflexionar y actuar sobre los problemas derivados de la realidad de la práctica(16). Además tiene la ventaja de estudiar los efectos de las acciones emprendidas, en la medida en que los participantes reflexionan acerca del proceso en forma permanente y evalúan los resultados del cambio para asegurarse que se han logrado los efectos esperados(17). Así mismo, favorece la interacción de los profesionales de la salud y los miembros de la comunidad involucrada en el proyecto y actúan como facilitadores de los procesos. Este método parte del principio de que toda comunidad posee los recursos para llevar a cabo su proceso de transformación(18).

\section{Participantes}

En la segunda y tercera fases del proyecto que aquí se presentan participaron 42 personas que viven en una vereda de 1.250 habitantes, de condición socioeconómica baja. Catorce de ellas en condición de discapacidad, con edades entre los 18 y 65 años y sus cuidadores familiares, seis miembros de la Junta de Acción Comunal, ocho líderes comunitarios y un equipo interdisciplinario de estudiantes y profesores investigadores de las facultades de Enfermería, Psicología y Administración de empresas y la promotora de salud designada por la Secretaría de Salud del municipio.

Consideración ética: Este estudio, aprobado por la Dirección de Investigación y el Comité de ética de la Universidad de La Sabana, fue también avalado por los participantes, convocados en una fase preliminar para la presentación del proyecto. En la reunión se les proporcionó información precisa, clara y oportuna acerca del objetivo del estudio y de sus diferentes fases, el método de recolección de la información, y el costo -beneficio del mismo. Posteriormente, se les solicitó en cada una de las fases que quienes estuvieran de acuerdo con participar firmaran un consentimiento informado, en el cual se aclaraba la confidencialidad y que podrían retirarse cuando así lo desearan. Para garantizar la privacidad de la información se modificaron los nombres de los participantes y de las personas citadas por ellos.

\section{Etapas del proyecto}

Primera fase de planeación: Se identificó el significado que tenía la discapacidad para las personas en esta condición, los cuidadores familiares y los miembros de la Junta de Acción Comunal de la vereda. La información se recopiló a través de entrevistas semiestructuradas de profundidad, grupos focales y un foro comunitario(19). Los resultados obtenidos en esta fase fueron analizados por los investigadores y confirmados por los participantes, quienes en un encuentro 
posterior identificaron la exclusión social como el problema prioritario, establecieron sus causas, identificaron los recursos disponibles en la comunidad y las estrategias para lograr la meta que según su percepción debía ser la inclusión social. La metáfora del árbol desarrollada por Tandon, Azelton y Stricklan(20), sirvió como estrategia para identificar a partir de cada una de sus partes un significado y una función. Así, el tronco representaba el problema principal, las raíces sus causas, las hojas los recursos disponibles y los mecanismos para obtener los resultados esperados. Por su parte, la metáfora del sol se utilizó también con un sentido práctico que permitía a los participantes seleccionar un símbolo para nominar el proyecto, que por su función permitiría nutrirlo, alimentarlo, darle vida y a la vez apropiarse de él. Con base en toda esta información los investigadores junto con la comunidad diseñaron un modelo de inclusión social fundamentado en la propuesta socioecológica de Urie Bronfrenbrenner(14). El modelo desarrollado incluyó 4 núcleos articuladores: 1. El núcleo de desarrollo personal y familiar, que busca potencializar las competencias de los participantes, que les permitan resolver los problemas cotidianos y a la vez impactar su desarrollo dentro de la comunidad, se desarrolló a través de tres estrategias proactivas (actividades) que incluyen el cuidado en casa, la formación de agentes de salud y las estrategias de apoyo a los cuidadores. 2. El núcleo de desarrollo comunitario trabaja para empoderar a la comunidad en el fortalecimiento de cada uno de sus miembros, y sus familias, en el desarrollo de estrategias centradas en la solución de problemas, en la implementación de un plan de acción y del seguimiento pertinente. Lo anterior se consolida a través de estrategias tales como: la identificación de recursos, la formación de líderes y los proyectos productivos. Las personas interesadas en consolidar las actividades productivas y el grupo de líderes, recibió un curso de microempresarios. 3. Las redes de bienestar están asociadas a la promoción de estilos de vida saludables que repercutirán en su productividad y que complementarán al núcleo de desarrollo personal y familiar. Este se nutre a través de tres estrategias proactivas como una forma de lograr el bienestar: las actividades lúdicas, la sensibilización y el apoyo en las creencias. Las actividades lúdicas se refieren a las estrategias encaminadas a forjar espacios de esparcimiento y recreación a nivel individual y grupal. Las actividades de inclusión y sensibilización buscan crear conciencia del rol que tienen todas las personas frente a la discapacidad y el reconocimiento del rol que deben cumplir estas personas en la sociedad, así como generar programas que favorezcan su inclusión a la comunidad(21). 4. La redes comunicativas, se parte de que el modelo de inclusión social no es un sistema cerrado sino que promueve la comunicación a través de cada uno de los subsistemas. Está diseñado para que los miembros estén conscientes de los servicios existentes y para animar a la comunidad a participar en las actividades. Igualmente, ayudan a que estas personas estén actualizando sus experiencias, accedan a la información de manera pertinente y oportuna para que puedan obtener respuesta a sus inquietudes y dificultades de una manera ágil y fluida, donde se informa a la comunidad a través de diversos medios sobre las actividades, los eventos y los logros obtenidos durante el desarrollo del programa.

Segunda fase de acción: Se inició con la implementación del programa y se continuaron los procesos de reflexión para establecer los avances y efectos del mismo sobre la problemática y la evaluación de los resultados alcanzados. Esta fase se caracterizó por el desarrollo de actividades conjuntas y procesos permanentes de reflexión y evaluación.

Tercera fase de evaluación: Se basó en las reflexiones de los participantes, los ajustes que se realizaron en el desarrollo de las fases anteriores y por último la percepción que tenían todos los actores frente a los resultados obtenidos, las sugerencias y recomendaciones. 


\section{Recolección y análisis de la información}

Durante la fase de acción, la información se recolectó a través de grupos de discusión con los participantes, los líderes comunitarios y el equipo de investigación. El propósito de estos grupos fue analizar la percepción que tenían cada uno de los actores acerca del programa, proponer estrategias para el fortalecimiento de cada uno de los núcleos articuladores y evaluar las acciones desarrolladas. Estas reuniones se constituyeron en una fuente muy importante de información para la reflexión y análisis de los avances del programa.

En la fase de evaluación se organizaron dos grupos focales, uno con los miembros de la junta de acción comunal, otro con el grupo de líderes de la comunidad que se consolidó para apoyar el programa y 11 entrevistas de profundidad semiestructuradas que se realizaron a las personas en condición de discapacidad que no tenían deficiencias de las funciones mentales y a los cuidadores.

La información recolectada en los grupos focales y en las entrevistas de profundidad fue grabada con autorización de los participantes y transcrita textualmente. Esta se complementó con el registro de las notas de campo que el grupo de investigadores tomó en el transcurso de las mismas, en las cuales se incluyeron las actitudes como tendencias a la acción, que dan cuenta de lo que las personas piensan, sienten $\mathrm{y}$ hacen frente a determinadas situaciones o temas de discusión, lo cual fue de gran utilidad para complementar la información obtenida.

Los investigadores al realizar las entrevistas de profundidad solicitaron a las personas en condición de discapacidad y a los cuidadores que describieran en forma amplia la percepción que ellos tenían acerca del programa, los logros alcanzados, así como las sugerencias y recomendaciones.

Posteriormente, se inició un proceso de análisis inductivo en el cual los investigadores realizaron una lectura cuidadosa de la transcripción de cada entrevista, con el propósito de familiarizarse con los datos. Se subrayaron cada una de las frases que reflejaban la vivencia de la situación, las necesidades sentidas y el apoyo social percibido por los participantes. Esta estrategia permitió la identificación de los códigos sustantivos, entendidos como la denominación que hace el investigador de los segmentos de los datos obtenidos de los participantes, que reflejan la opinión de los participantes con relación a los resultados obtenidos con el programa de participación comunitaria. Finalmente, se realizó una agrupación de los códigos en categorías, que fueron definidas con base en las interpretaciones analíticas(22).

\section{RESULTADOS}

En total se identificaron 23 códigos sustantivos que representan las expresiones utilizadas por los entrevistados y 10 categorías. Algunos de estos códigos se encontraron únicamente en las personas con discapacidad, otros sólo en los cuidadores y otros en los grupos de líderes comunitarios. Vale la pena anotar que algunos son comunes a todos (ver Tabla 1).

Tabla 1: Códigos sustantivos y categorías

\begin{tabular}{|c|c|c|c|}
\hline & Códigos Sustantivos & Categorías & Definición Categoría \\
\hline \multirow[t]{2}{*}{$\begin{array}{l}\text { Personas en } \\
\text { condición de } \\
\text { discapacidad }\end{array}$} & $\begin{array}{l}\text { Mejoría del estado de } \\
\text { salud } \\
\text { Motivación } \\
\text { Productividad }\end{array}$ & Bienestar & $\begin{array}{l}\text { Percepción de satisfacción personal } \\
\text { relacionada con el estado de salud y con los } \\
\text { logros alcanzados }\end{array}$ \\
\hline & $\begin{array}{l}\text { Satisfacción de } \\
\text { necesidades } \\
\text { Capacitación }\end{array}$ & Respaldo & $\begin{array}{l}\text { Es el apoyo de tipo familiar, comunitario e } \\
\text { institucional que reciben las personas en } \\
\text { condición de discapacidad para satisfacer sus } \\
\text { necesidades }\end{array}$ \\
\hline
\end{tabular}




\begin{tabular}{|c|c|c|c|}
\hline & Necesidad de apoyo & $\begin{array}{l}\text { Dependencia del } \\
\text { programa }\end{array}$ & $\begin{array}{l}\text { Necesidad de recibir ayuda institucional y de la } \\
\text { comunidad para alcanzar sus fines y propósitos }\end{array}$ \\
\hline & $\begin{array}{l}\text { Aislamiento } \\
\text { Abandono }\end{array}$ & Exclusión Social & $\begin{array}{l}\text { Percepción de abandono por parte de la } \\
\text { comunidad }\end{array}$ \\
\hline \multirow[t]{2}{*}{$\begin{array}{l}\text { Cuidadores } \\
\text { familiares }\end{array}$} & $\begin{array}{l}\text { Compañía } \\
\text { Satisfacción de } \\
\text { necesidades }\end{array}$ & $\begin{array}{l}\text { Reconocimiento } \\
\text { del otro }\end{array}$ & $\begin{array}{l}\text { Sentimiento de gratitud con la comunidad y } \\
\text { con las instituciones por haber identificado y } \\
\text { contribuido en la satisfacción de las } \\
\text { necesidades personales y familiares }\end{array}$ \\
\hline & $\begin{array}{l}\text { Orientación } \\
\text { Relevo } \\
\text { Reconocimiento programa } \\
\text { Alcaldía }\end{array}$ & Respaldo & $\begin{array}{l}\text { Es el apoyo de tipo comunitario e institucional } \\
\text { que reciben los cuidadores familiares para } \\
\text { realizar sus labores de cuidado y satisfacer sus } \\
\text { necesidades. }\end{array}$ \\
\hline \multirow[t]{2}{*}{$\begin{array}{l}\text { Líderes de la } \\
\text { comunidad }\end{array}$} & $\begin{array}{l}\text { Compromiso } \\
\text { Identificación de } \\
\text { necesidades } \\
\text { Fortalecimiento del } \\
\text { vínculo } \\
\text { Reconocimiento del otro } \\
\text { Disposición para actuar }\end{array}$ & Sensibilización & $\begin{array}{l}\text { Efecto de reconocer el valor y las necesidades } \\
\text { de las personas en condición de discapacidad y } \\
\text { sus familias y disposición e interés para } \\
\text { apoyarlos en su desarrollo personal y familiar }\end{array}$ \\
\hline & $\begin{array}{l}\text { Compromiso } \\
\text { Recursos de apoyo }\end{array}$ & Apoyo social & $\begin{array}{l}\text { Capacidad de ofrecer respaldo personal, } \\
\text { comunitario y familiar a las personas con } \\
\text { discapacidad y sus familias. }\end{array}$ \\
\hline $\begin{array}{l}\text { Líderes Junta de } \\
\text { Acción Comunal }\end{array}$ & $\begin{array}{l}\text { Interés } \\
\text { Compromiso } \\
\text { Necesidad de organización }\end{array}$ & Sensibilización & $\begin{array}{l}\text { Efecto de reconocer el valor y las necesidades } \\
\text { de las personas en condición de discapacidad y } \\
\text { sus familias y disposición e interés para } \\
\text { apoyarlos en su desarrollo personal y familiar }\end{array}$ \\
\hline
\end{tabular}

Los resultados igualmente se analizan en términos de las actividades desarrolladas en cada uno de los núcleos articuladores y la percepción de los participantes relacionada con cada uno de ellos. En el proceso de análisis se realizó una nueva lectura de los códigos y categorías identificados y se organizaron en cada uno de los núcleos como puede apreciarse en la Tabla 2.

Tabla 2 Categorías según núcleos articuladores

\begin{tabular}{|c|c|}
\hline Núcleos articuladores & Categorías \\
\hline Desarrollo personal y familiar & $\begin{array}{c}\text { Bienestar } \\
\text { Respaldo } \\
\text { Exclusión }\end{array}$ \\
\hline Desarrollo comunitario & $\begin{array}{c}\text { Dependeno social } \\
\text { Apoyo }\end{array}$ \\
& $\begin{array}{c}\text { Satisfacción de necesidades } \\
\text { Recursos } \\
\text { Bienestar } \\
\text { Dependencia institucional }\end{array}$ \\
\hline Redes de bienestar & $\begin{array}{c}\text { Reconocimiento del otro } \\
\text { Apoyo social } \\
\text { Sensibilización }\end{array}$ \\
\hline Redes de comunicación & Sensibilización \\
\hline
\end{tabular}




\section{Núcleo de desarrollo personal y familiar:}

Los avances obtenidos en este núcleo se aprecian en las expresiones de los participantes durante las entrevistas de profundidad y grupos focales, de las cuales se identificaron los códigos sustantivos y se organizaron en categorías que reflejan la percepción de los participantes. Algunas de éstas son:

\section{Bienestar}

Esta categoría está representada en los siguientes códigos: Mejoría del estado de salud, motivación, relevo y respaldo.

La mejoría del estado de salud se aprecia en la siguiente expresión de una mujer de 60 años, quien manifestaba: "Sí señora eso es muy bueno, yo también aprendí mucho, yo estaba casi entumida.... (silencio) no, de verdad (afirmando con la cabeza), no entumida completamente, eso sí, caminaba y eso si era que casi no alcanzaba... y entonces los estudiantes me dijeron, me dijeron eso de los ejercicios y caminar en las puntas de los pies (se levanta de la silla y hace la demostración), si así 20 veces y yo de todo eso hice, pues ahora aunque todavía me duele mucho (señala sus piernas) claro, pero mire doctora ya me desentumí completamente (afirma con la expresión)".

La motivación se encuentra en expresiones de los cuidadores como ésta: "Cuando venía, ella lo hacía reír (se sonríe) y lo estimulaba... le hacía ejercicios. Es un programa muy bueno (afirma con la expresión) porque vienen y le toman la tensión a ella y la sacan a caminar y nos toman la tensión a juntas".

Sentirse productivo es un estado que produce sentimientos de bienestar: "No me gusta martillar porque no puedo, porque mis brazos no me dejan, entonces como a mí me gusta estar aquí, entonces me pusieron a contar las mechitas de las escobas".

\section{Respaldo}

El respaldo se relaciona con la satisfacción de necesidades como se aprecia en la siguiente expresión de una persona en condición de discapacidad: "Yo veo que nos hemos beneficiado harto, porque antes no tenía el apoyo de una droga, de una pomada, todos nos hemos beneficiado y el que diga lo contrario pues estaría mintiendo (expresión de afirmación)".

También se evidencia en la preparación recibida: "Las niñas me enseñaron a hacer la curación, para que los días que no vinieran ellas, yo la pudiera hacer, ellos le explican a uno su dieta, qué hacer de comer, todo eso".

Del mismo modo se puede apreciar en la percepción de relevo o alivio. Una señora de 45 años quien tiene que cuidar a tres familiares en condición de discapacidad manifestaba: "Pues ahora que ellas se han quedado con ellos, si ha sido una ayuda porque me ha tocado salir a hacer mis cosas, pero no me demoro (expresión de negación)... tan solo un poquito".

Los líderes de la comunidad se han comprometido con éste respaldo como se evidencia en ésta expresión: "Hicimos el recorrido por la vereda, donde doña Elisa, Clara y nos devolvimos acá, me tomaron la tensión y me preguntaron cómo me había parecido, que si estaba cansada... y yo sí les dije que quería volver a salir a caminar otra vez"

Al analizar los códigos sustantivos que respaldan estas categorías, se encontraron la dependencia del programa y la exclusión que deben evaluarse con detenimiento para realizar los ajustes correspondientes:

\section{Dependencia del programa}

La dependencia del programa se evidencia en frases como la de esta persona en condición de discapacidad: "Cuando ellos no vienen siento que voy de para atrás, que sea la voluntad de Dios", "Esperemos que lleguen pronto a ver si me ayudan.....sería muy bueno" (énfasis en la expresión). Lo anterior muestra la manera como la interacción entre los miembros de la comunidad y los estudiantes participantes genera vínculos cercanos y necesarios durante la implementación del programa, lo cual está 
asociado a la posibilidad de que en su presencia es posible resolver sus dificultades. En este sentido, se hace evidente la implementación de estrategias que permitan a los miembros ganar en autonomía.

\section{Exclusión:}

La exclusión se ve reflejada en el aislamiento, así lo expresa un hombre de 67 años: "Ya no me relaciono mucho con la comunidad, antes sí andaba para arriba y pa' bajo, pa' un lado y otro, ahora casi no salgo por ahí a caminar". También se observa en los sentimientos de abandono "no, de la comunidad?... muy poco, de los amigos pero también es muy poco... no han vuelto, antes cuando estaba bien, pues todo el mundo era amigo, ahora cuando está mal no ... es muy poco (con expresión de tristeza)".

\section{Núcleo de desarrollo comunitario}

El trabajo, a partir de este núcleo, permite dar cuenta del impacto de las coaliciones que han sido posibles gracias a las intervenciones en los otros núcleos articuladores. Esto se refleja en el empoderamiento de la comunidad para facilitar las condiciones que permiten resolver las problemáticas enfrentadas por las personas y sus familias y promover la búsqueda de soluciones asertivas y reactivas, lo que significa que la autorregulación entendida como la máxima expresión de la autonomía de una comunidad se hace evidente en el empoderamiento de la misma. Desde aquí se verifica si se están utilizando los insumos proporcionados y que han sido asumidos como propios y dan cuenta de lo que está ocurriendo dentro del sistema. Los lineamientos dados en el curso motivaron a los participantes a organizar una microempresa de escobas y traperos y a complementar esta actividad con otras de apoyo para las personas en condición de discapacidad. Así relata uno de los líderes este proceso: "Hemos colaborado siempre, se comenzó con las escobas, luego se hizo un bingo, luego una subasta de ropa de segunda, luego se abrió una cuenta en el banco, se sacó una plata de allá y se hizo el grupo de escobas"

En este núcleo se evidencian las siguientes categorías:

\section{Apoyo social:}

La categoría de apoyo social a las personas en condición de discapacidad se evidencia en esta expresión, que refleja el compromiso de uno de los líderes comunitarios: "Tenemos que tratar de ayudar a los demás, así no les demos nada, pero démosles ese amor que ellos necesitan".

El compromiso es evidente en expresiones como ésta de una de las líderes, al referirse al taller de escobas: "Yo creo que mis días, me den un alientito de vida, para estar con ustedes en las buenas y en las malas, pero quiero que surjamos, que no nos quedemos pensando que no lo podemos hacer nosotros, que no nos dejemos vencer".

También en la necesidad de identificar recursos de apoyo como se aprecia en esta expresión: "Podemos seguir sacando como un fondito para los discapacitados... para darles lo que ellos más o menos necesitan, porque no tenemos para todo, pero sí podemos ayudar con una primita, con una platica para que compren una ropita, eso se les dio el año pasado".

El compromiso fue evidente en el grupo focal con los miembros de la Junta de acción comunal, uno de ellos manifestaba: "Sería bueno poder seguir con el programa con la organización de un cronograma... De un comité de discapacidad...que se estuviera mirando el programa mensualmente".

\section{Satisfacción de necesidades}

Los líderes expresan compromiso para tratar de satisfacer las necesidades: "Puede uno ver que necesitan, que vamos a ir a visitarlos, todas esas cosas son muy importantes y que la gente se dio cuenta que se dejan tratar y que son útiles".

Uno de los miembros de la Junta de ac- 
ción comunal expresaba: "Es necesario que tengamos una estadística y que el programa se vincule al de la alcaldía. También se necesita involucrar más a la comunidad y hacerles entender la necesidad. Hacerles conocer los medios para que las personas se puedan desplazar sin dificultad. Por eso manejar la estadística, tener un grupo terapéutico y un alivio para las personas".

\section{Bienestar}

El bienestar se expresa así: "A mí me ha motivado la compañía de los otros", otra persona manifestaba "A mí me gusta porque me distraigo.... Es que a veces me siento aburrida".

\section{Dependencia institucional}

En expresiones como las que se presentan a continuación se evidencia que, aunque las personas se sienten satisfechas, aún persiste dependencia institucional, "Ellas (refiriéndose a las estudiantes) lo consienten mucho a uno y cuando ellas se van siente uno el vacío... siente uno eso de que ya vienen ellas... esperemos que lleguen pronto a ver si me ayudan".

\section{Núcleo de redes de bienestar}

$\mathrm{Al}$ analizar las expresiones encontradas en las entrevistas se identifican los siguientes códigos y categorías asociadas con éste núcleo:

El reconocimiento del otro se aprecia en la expresión de la mamá de un muchacho de 20 años con retardo mental moderado cuando manifestaba: "El programa me ha gustado, porque ahora a mi niño lo saludan, no lo insultan ni lo golpean, lo tratan bien, lo tienen en cuenta... (énfasis en la expresión)".

También en este comentario de una líder comunitaria "que antes uno era como indiferente con ellos ( con las personas con discapacidad) porque no llegaba quizás uno a pensar que era una persona común y corriente como uno, uno lo veía como debe estar allá (señala un lugar apartado) y yo acá... hoy no (énfasis en la expresión), hoy uno sabe que esa persona necesita de uno, que se va a subir a un carro que ayúdele, que se necesita un médico, que cómo está comiendo... entonces uno se siente que está siendo útil para las otras personas".

\section{Núcleo de redes comunicativas}

Es importante destacar los procesos que se evidenciaron en relación con este núcleo:

Sensibilización: Se muestra que se ha iniciado un proceso de concientización de las personas frente a ésta problemática, esto es evidente en la expresión de uno de los miembros de la Junta de Acción: "Ha habido un logro real que es crear la conciencia de la gente, casi nadie hablaba de la discapacidad, en este momento se ha creado la conciencia, que son personas con limitaciones..., (niega con la expresión), que no son una carga...”.

Al realizar un análisis global, se observa que las personas que han estado involucradas directamente en el proyecto reconocen los resultados positivos que ha tenido el programa y la necesidad de incluirlo como un eje de desarrollo de la comunidad. Para lograrlo es indispensable continuar el proceso de sensibilización y empoderamiento de todas las personas frente a la inclusión social, la consecución de recursos propios y la articulación con el gobierno del municipio.

\section{DISCUSIÓN}

Los resultados presentados muestran cómo la inclusión social es un fenómeno que debe ser entendido desde la perspectiva de proceso y de resultado. En el primer caso, se observa que es importante establecer como punto de partida los elementos que hacen posible la inclusión. Estos elementos están directamente relacionados con los recursos tanto personales como comunitarios, los cuales al potencializarse a través de actividades de participación comunitaria posibilitan en el tiempo transformaciones 
que buscan minimizar la exclusión. En este sentido todos los núcleos del modelo socioecológico aquí propuesto, de desarrollo personal y familiar, de desarrollo comunitario, de redes de bienestar y de comunicación, están en disposición y a la vez en interacción con cada uno de los subsistemas a nivel individual y familiar, comunitario e institucional, para ir logrando cambios que se retroalimentan y se modifican de acuerdo con las necesidades.

Lo anterior es posible en la medida en que se favorecen desde la interdisciplinariedad, los procesos de acompañamiento para la formación y empoderamiento de la comunidad para participar en actividades como el cuidado en casa y la formación de agentes de salud a través de las cuales se generan las condiciones para que la autonomía se privilegie. De esta manera se ha logrado incrementar el apoyo social, y para que las personas en condición de discapacidad y las familias puedan afrontar los problemas que se presentan en la cotidianidad.

Frente a esta realidad, al analizar las respuestas de los participantes a la pregunta relacionada con la percepción que tienen sobre el proceso de desarrollo del programa se evidencian sentimientos de bienestar y respaldo. Este bienestar se asocia con la mejoría de su estado de salud en la medida en que las tareas desarrolladas con los asesores de la Universidad son valoradas por su efecto a corto plazo, y se aprecia en la posibilidad de realizar actividades por y para ellos mismos, tales como la disminución del dolor y la oportunidad de acceso a otras actividades que antes no eran posibles. El aprendizaje aquí es un fenómeno que ha sido reconocido en múltiples programas de atención a las personas en situación de discapacidad, en los cuales el objetivo es reducir la severidad de una condición como en la investigación llevada a cabo por Rasvesboot Seekins y Lingrem(23), en la cual los participantes que tenían problemas de movilidad sentían que los días de malestar eran cada vez menores.

En resumen, el trabajo alrededor de la identificación de aquellos aspectos que fortalecen el desarrollo, esperando que este conocimiento pueda ser usado para optimizar el funcionamiento de las personas y las familias, ha sido una perspectiva que busca identificar y trabajar sobre todas aquellas influencias psicosociales que afectan a la persona, incluyendo tanto los aspectos internos como externos $(24,25,26)$.

Por otro lado, la percepción de respaldo vinculada también con el bienestar y con la presencia del otro, se consolida en una apreciación muy importante, dado que en su historia no disponían de la posibilidad de recibir de la comunidad atención a sus necesidades asociadas a su discapacidad. Las expresiones que se relacionan con esta percepción de respaldo se evidencian en afirmaciones que comparan el presente con el pasado, en cuanto a que "antes no tenía para... y hoy.... Este respaldo es también percibido por los cuidadores, quienes reportan alivio en sus funciones a través de actividades de relevo. En esta misma dirección, los líderes de la comunidad han asumido un compromiso que está presente en las decisiones y en las acciones por hacer de su participación en este programa un reto, que vincule cada vez más a otros miembros para lograr reducir la desesperanza de estas personas. Es importante destacar en las expresiones de los líderes comunitarios actitudes y sentimientos positivos al manifestar su reconocimiento frente a las necesidades y su disposición de ayuda, así como la motivación por persistir en este esfuerzo mediante la consolidación de redes formales. Es aquí donde se evidencia la reciprocidad que existe en la interacción de los diferentes sistemas.

Si bien lo anterior muestra como a través del proceso se van obteniendo logros importantes que se espera en el tiempo sigan su curso y se asuman como propuestas colaborativas de interdependencia y de formación de redes, el proceso de construcción de comunidad para lograr la tan ansiada autonomía propia de la inclusión social, debe tomarse con cierta reserva en la medida en que en la evaluación siguen persistiendo expresiones que connotan la necesidad de la presencia del otro, desde el vínculo formado en la interacción.

Otro aspecto complementario del análisis 
anterior, está relacionado con la persistencia de la percepción de exclusión, producto de su condición de discapacidad. Algunos participantes asocian su condición actual como la responsable del cambio en algunos actores de su historia. Se crea un sentimiento negativo cuando en la reflexión sobre la pérdida de sus funciones, significa también la pérdida de posibilidades. De hecho expresaron "Antes cuando estaba bien me visitaban, me invitaban pero ahora estoy en el olvido".

Estos componentes emergentes de la valoración del programa piloto, como son la dependencia del programa y la percepción de exclusión, deberán ser considerados a futuro en el curso de las propuestas de intervención desarrolladas conjuntamente con la comunidad, ya que al ser previstas con antelación como posibles efectos derivados de este trabajo, se puedan contemplar para optimizar los resultados y contribuir de manera explícita al desarrollo.

El núcleo de desarrollo comunitario que incluyó la implementación de estrategias para la formación de líderes y proyectos productivos con el apoyo de la participación interdisciplinaria, es una realidad con la cual el programa se construyó y del cual son muy positivos los alcances en el mediano plazo. Se observa la constitución de una microempresa, en la cual la participación de las personas en situación de discapacidad, sus familias y los líderes comunitarios es la expresión de que es posible desde una propuesta de trabajo socioecológico establecer las vinculaciones y las relaciones necesarias para lograr como resultado la inclusión social. Este producto tiene la fortaleza de contar con el aporte de las potencialidades de cada uno de los participantes, que en su conjunto nutren el trabajo y emulan a otros la expectativa de que la participación comunitaria es el eje sobre el cual se pueden tejer otras posibilidades, que en el pasado eran pensadas sólo para personas que no presentan discapacidad.

La sensibilización frente a la discapacidad, fue otro resultado percibido especialmente en la junta de acción comunal. En la evaluación sobre la percepción del programa, se detectan opiniones muy diversas, pero que en conjunto reflejan la necesidad de tomar como punto de partida esta experiencia. Se discute la necesidad de gestionar desde su competencia un Comité de Discapacidad, con un cronograma que dé posibilidad de lograr otras metas y verificar a través del tiempo su eficacia.

Finalmente, los logros relativos al desarrollo personal, familiar y comunitario fueron posibles en la medida en que la comunidad se vinculó de manera activa a la construcción de un modelo orientado por el equipo interdisciplinario y sustentado en los principios de desarrollo propuestos por Bronfrenbrenner(14). El fortalecimiento de los núcleos de bienestar y comunicación, tan importantes dentro de esta propuesta, se consideran el telón de fondo para acompasar y viabilizar todas las acciones que buscan la inclusión social.

En un proceso de éstas características la construcción participativa del modelo fue fundamental para reconocer el valor y las potencialidades de las personas en condición de discapacidad, reconocer las necesidades de los cuidadores familiares e identificar las posibilidades que tiene la comunidad para asumir la responsabilidad y el compromiso de apoyar a quienes más lo necesitan en su proceso de desarrollo personal y familiar. Se crea desde este trabajo un estilo de análisis frente a los problemas, que asegura en el devenir de la vida de cada uno de los participantes, una posibilidad de actuación que busque su solución desde los recursos individuales y comunitarios.

\section{REFERENCIAS}

1. Departamento Administrativo Nacional de Estadística (DANE). Censo Nacional de Población, 2005. Disponible en http:// www.dane.gov.co/censo/files/discapacidad/ discapacidad_mundo.pdf [Extraído el 10 de Julio 2007]

2. Gender,A R Scope of rehabilitation and 
rehabilitation nursing. En: Chin P, Finocchiaro D. Rosebrough, A. Rehabilitation nursing practice. New York:Mc Graw-Hill, first edition, 1996.

3. Cuervo, C, Escobar M y Trujillo A. Más allá de una visión clínica de la discapacidad. Revista Ocupación Humana. 2000; 8 (4): 12-16.

4. Céspedes G. La nueva cultura de la discapacidad y los modelos de rehabilitación. Aquichan. 2005; 5(1): 108-113

5. Zabriskei R, Lundberg, N.R. y Groff, D.G (2004). Quality of Life and Identity: Benefits of a community - based therapeutic recreation and adaptive sport program. Therapeutic Recreation Journal. 2004; 39 (3): 176-191.

6. Zabriskei R, Heine L. A refocus on Family. Therapeutic Recreation Journal. 2003; 37 (1): 15-17.

7. Moreno M. Políticas y concepciones en discapacidad: un binomio por explorar. Bogotá: Universidad Nacional. 2007.

8. Moreno M. Inclusión de las personas con Discapacidad: reflexiones, realidades y retos. Universidad Nacional de Colombia. 2004.

9. Wheeler S; Lane S, McMahon B. Community Participation and Life Satisfaction Following Intensive, Community-Based Rehabilitation Using a Life Skills Training Approach. Occupation, Participation and Health. 2007; 27 (1): 13-22.

10. Oliver M. Understanding disability from theory to practice. London: Macmillan. 1996.

11.Márquez A. Rehabilitación Basada en la Comunidad en la Costa Pacífica. Revista Fundamento. 2002; 13: 44-53.

12.Vitztum-Komanecki J M. Community integration and inclusion: Developmental outcomes for preschoolers with disabilities in inclusive settings. Disertación, Universidad de Indiana. 2007.

13.Blessing L A. Social integration, support and sense of community: Exploring the productive activities of persons with a cognitive disability. Queen's University at
Kingston (Canada), 2004. Disponible en http://proquest.umi.com/pqdweb?did $=8$ $85630241 \&$ sid $=5 \&$ Fmt $=2 \&$ client $I d=4671$ 8. Extraído el 8 de Marzo, 2008].

14.Bronfrenbrenner $\mathrm{U}$. The Ecology of $\mathrm{Hu}-$ man Development. Cambridge:

University Press. 1979.

15.Schuller N. Disabled People, Crime and Social Inclusion. Community Journal. 2005; 4:4-13.

16.Freeman E M. Exemplar: Practical discourse as action research: Inquiry into post- Myocardial Behavioral Coaching. En: Munhall P (Ed.), Nursing Research a Qualitative Perspective. Boston, Massachusetts: Jones \& Bartlett Publishers. 2007.

17.Streuber H J, Carpenter H. Qualitative Research in Nursing Advancing the Human Imperative. Philadelphia: Lippincott 2nd edition. 1999.

18.Martín A. Psicología Social Comunitaria. Madrid: Pirámide. 2002.

19.Moreno M E, Rodríguez M C M, Gutiérrez M, Ramírez L, Barrera O P. ¿Qué significa la discapacidad? Aquichán. 2006; 6(1):7891.

20.Tandon K, Azelton K, Strickland L. Constructing a Tree for Community leaders: contexts and processes in collaborative inquiry. American Journal of Community Psychology. 1998; 26( 4):669-696.

21.Rodríguez M C, Alvarado A M, Moreno M E. Construcción participativa de un modelo socioecológico de inclusión social para personas en situación de discapacidad. Acta Colombiana de Psicología. 2007; 10 (2):181-187.

22.Charmaz K. Constructing grounded theory. A practical guide through qualitative Analysis. Londres: Sage Publications. 2006: 43-45.

23.Rasvesloot C H, Seekins T, Lingren S. Health promotion for people with disabilities: development and evaluation of living well a disability program. Health Education Research. 2007;4:522-532.

24.Bubolz M M, Santag M S. Human Ecology 
Theory. En: P.G. Bouss, W, J Doher, R, La Rosa W, Shumn \& S. K Stein Metz (Eds) Sourcebook of family theories and methods: A contextual approach. Nueva York: Plenum Press. 1993.

25.La Vigna G W, Willis T J. A Positive
Behavioral Support Model for Breaking the Barries to Social and Community Inclusion. Learning Disability Review.2005;10:16-23. 26.Mertensmeyer C, Fine M. Parentlink: A model of Integration and support for. 2000. 